\title{
Asymmetrical Polar Modification of a Bivanadium-Capped Keggin POM by Multiple Cu-N Coordination Polymeric Chains
}

Jing-quan Sha, ${ }^{[\mathrm{a}, \mathrm{b}]}$ Jun Peng, ${ }^{*[\mathrm{a}]}$ Hong-sheng Liu, ${ }^{[\mathrm{a}]}$ Jing Chen, ${ }^{[\mathrm{a}]}$ Ai-xiang Tian, ${ }^{[\mathrm{a}]}$ Peng-peng Zhang, ${ }^{[\mathrm{a}]}$ and Zhongmin $\mathrm{Su}^{*[\mathrm{a}]}$

${ }^{a}$ Key Laboratory of Polyoxometalate Science of Ministry of Education, Faculty of Chemistry, Northeast Normal University, Changchun, Jilin, 130024, P. R. China

E-mail: jpeng@nenu.edu.cn or pjun56@yahoo.com

${ }^{\mathrm{b}}$ Faculty of Chemistry and Pharmacy, Jiamusi University, Jiamusi, HeilongJiang, 154007, P. R. China 
Fig.S1 Ball and stick representation of the $\mathrm{Cu}{ }^{\cdots} \mathrm{Cu}{ }^{\prime \cdots} \mathrm{Cu}$ chains.

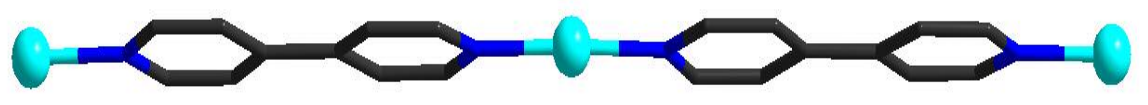

Fig.S2 Combined polyhedral/wire representation of chain structure in $\mathbf{1}$, the $\left\{\mathrm{AsMo}_{12} \mathrm{~V}_{2}\right\}$ clusters as pendants of the $\mathrm{Cu}($ bipy) chains projecting towards two sides.

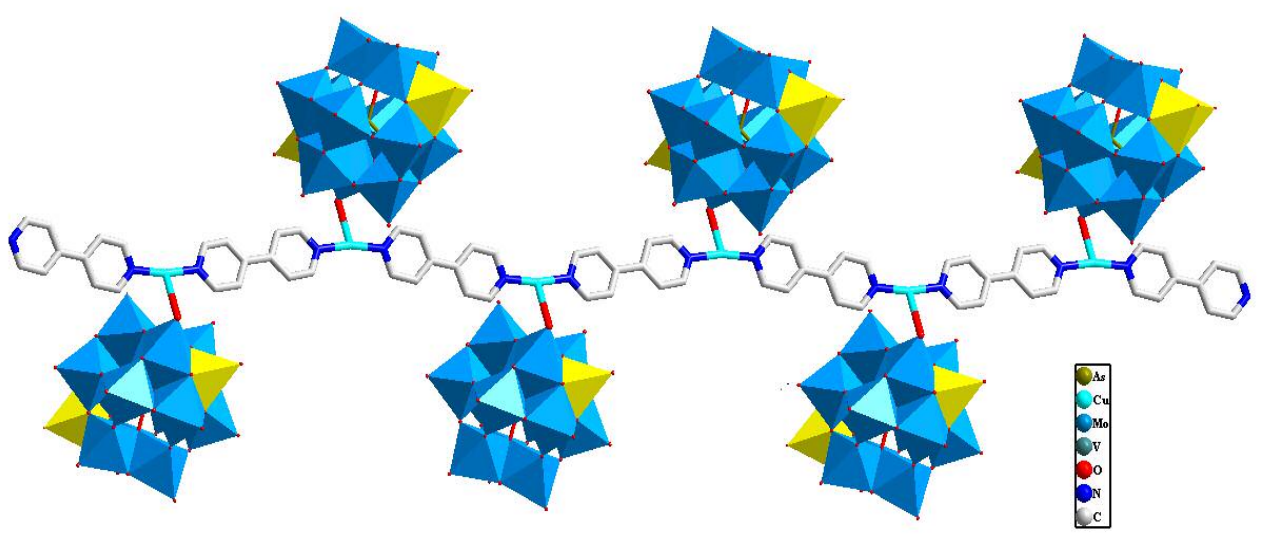

Fig.S3 Combined polyhedral/wire representation of "raillike" double monotrack Cu-bpe chains modified POMs, in which POMs array in the "side by side " mode.

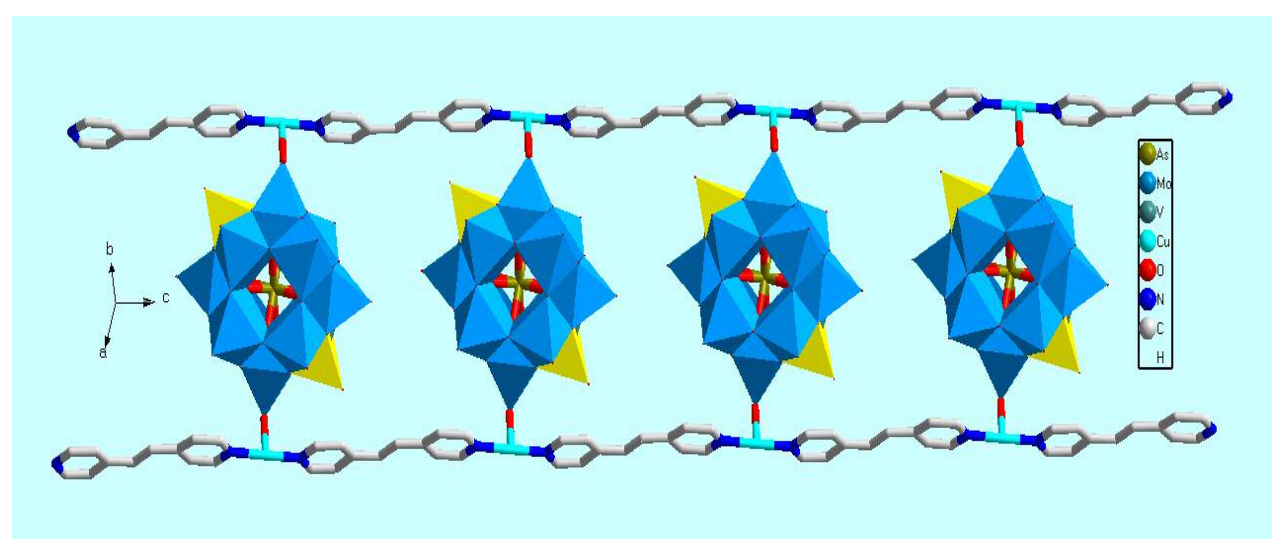


Fig.S4 The structure view showing intermolecular short interaction and offset face-to-face $\pi \cdots \pi$ stacking between the linear $\mathrm{Cu}_{2}$-bipy chains and two adjacent "rails"

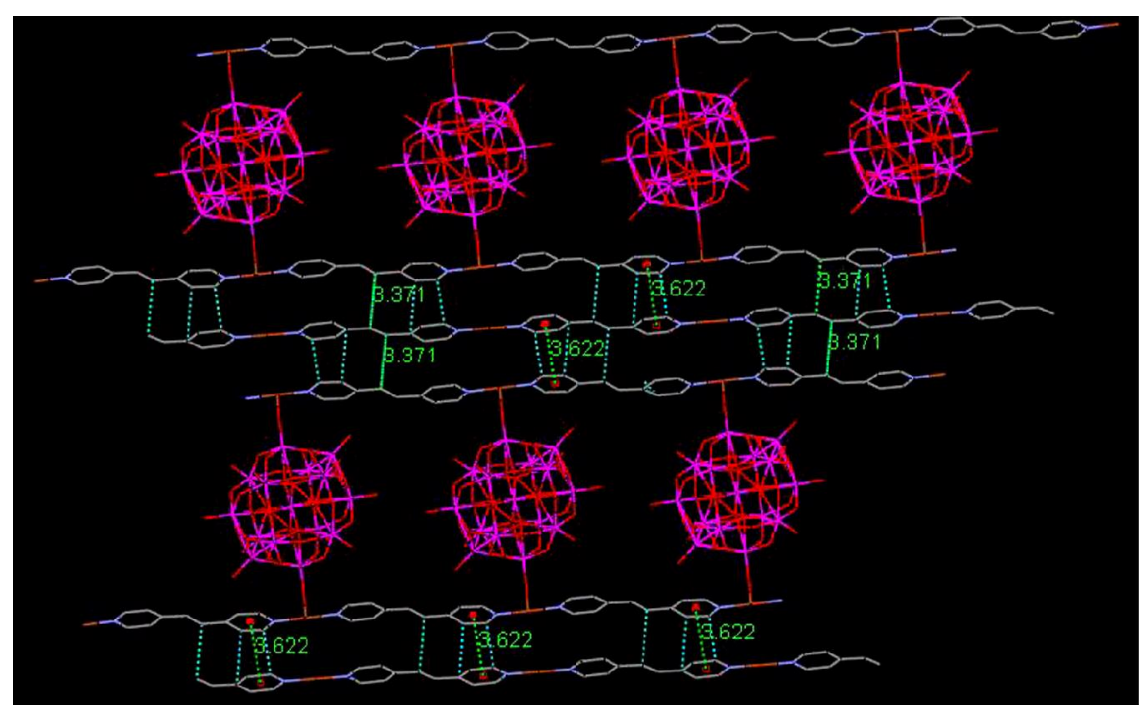

Fig.S5 The structure view showing intermolecular short interaction between the bpe molecule and the $\left\{\mathrm{AsMo}_{12} \mathrm{~V}_{2}\right\}^{\prime}$ cluster in $\mathbf{2}$.

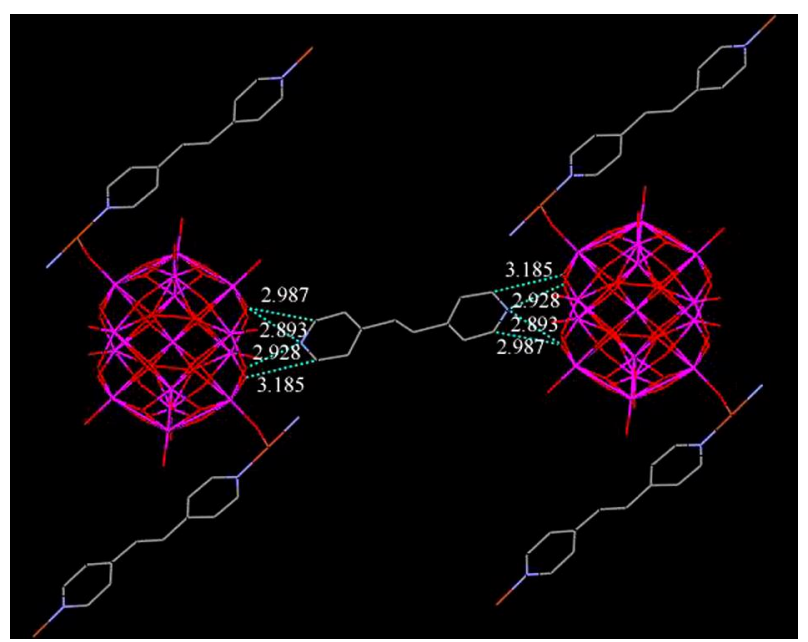

Fig.S6 The IR spectra of compounds 1 and $\mathbf{2}$.

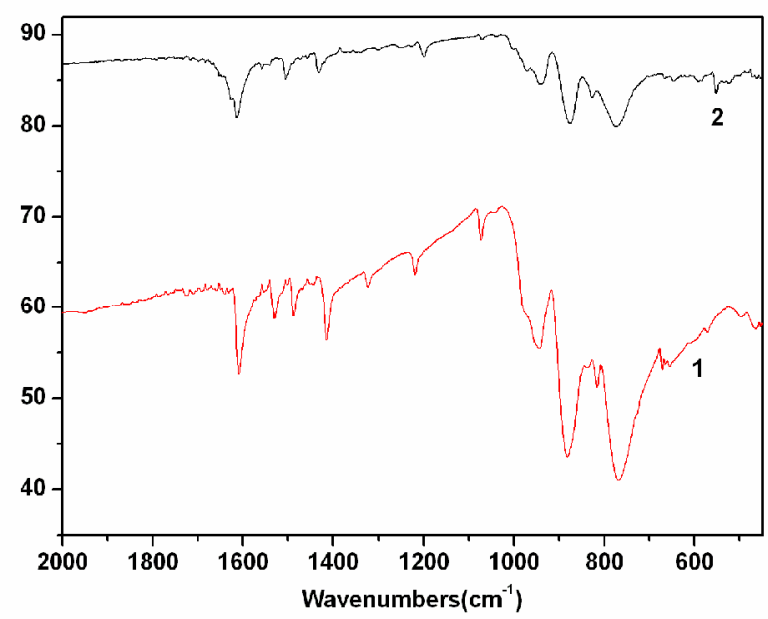


Fig.S7 The XPS of compounds 1 and 2.
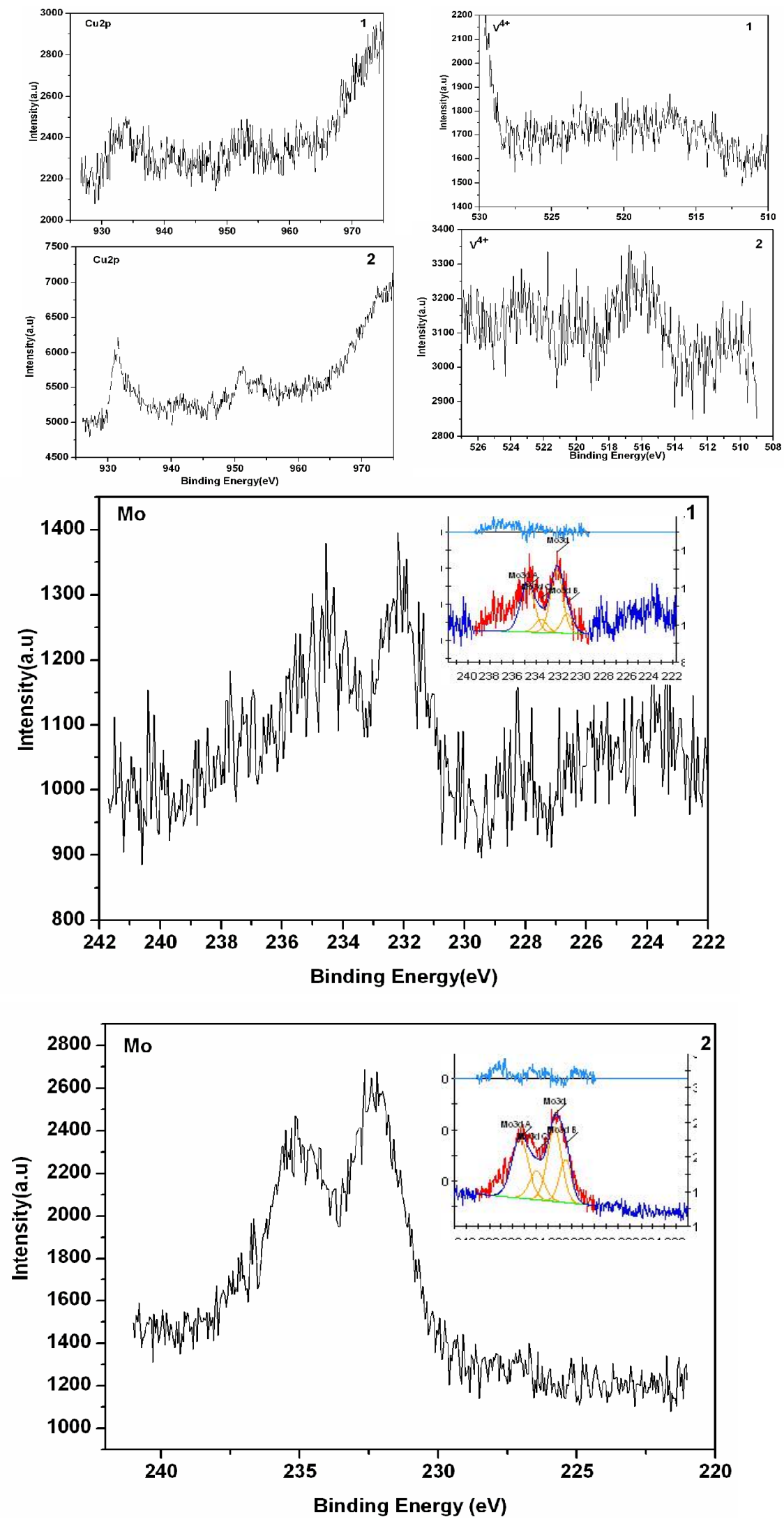
Table S1 Selected bond lengths $(\AA)$ and bond angles (deg) for compound $\mathbf{1}$.

\begin{tabular}{|c|c|c|c|}
\hline $\operatorname{As}(1)-\mathrm{O}(30)$ & $1.635(6)$ & $\mathrm{Mo}(6)-\mathrm{O}(16)$ & $1.719(6)$ \\
\hline $\operatorname{As}(1)-\mathrm{O}(65)$ & $1.656(6)$ & $\mathrm{Mo}(6)-\mathrm{O}(46)$ & $1.828(6)$ \\
\hline $\operatorname{As}(1)-\mathrm{O}(01)$ & $1.666(6)$ & $\mathrm{Mo}(6)-\mathrm{O}(54)$ & $1.864(6)$ \\
\hline $\mathrm{As}(1)-\mathrm{O}(31)$ & $1.671(6)$ & $\mathrm{Mo}(6)-\mathrm{O}(43)$ & $2.014(6)$ \\
\hline $\mathrm{Cu}(1)-\mathrm{N}(8)$ & $1.905(8)$ & $\mathrm{Mo}(6)-\mathrm{O}(55)$ & $2.036(6)$ \\
\hline $\mathrm{Cu}(1)-\mathrm{N}(9)$ & $1.910(8)$ & $\mathrm{Mo}(6)-\mathrm{O}(31)$ & $2.376(6)$ \\
\hline $\mathrm{Cu}(1)-\mathrm{O}(45) \# 1$ & $2.395(6)$ & $\mathrm{Mo}(7)-\mathrm{O}(40)$ & $1.707(6)$ \\
\hline $\mathrm{Cu}(2)-\mathrm{N}(4)$ & $1.889(8)$ & $\mathrm{Mo}(7)-\mathrm{O}(68)$ & $1.846(6)$ \\
\hline $\mathrm{Cu}(2)-\mathrm{N}(10)$ & $1.904(8)$ & $\mathrm{Mo}(7)-\mathrm{O}(72)$ & $1.860(6)$ \\
\hline $\mathrm{Cu}(2)-\mathrm{O}(52)$ & $2.252(7)$ & $\mathrm{Mo}(7)-\mathrm{O}(61)$ & $1.991(6)$ \\
\hline $\mathrm{Cu}(3)-\mathrm{N}(7)$ & $1.913(8)$ & $\mathrm{Mo}(7)-\mathrm{O}(74)$ & $2.079(6)$ \\
\hline $\mathrm{Cu}(3)-\mathrm{N}(6)$ & $1.928(8)$ & $\mathrm{Mo}(7)-\mathrm{O}(01)$ & $2.389(6)$ \\
\hline $\mathrm{Cu}(3)-\mathrm{O}(42)$ & $2.312(6)$ & $\mathrm{Mo}(8)-\mathrm{O}(9)$ & $1.685(6)$ \\
\hline $\mathrm{Cu}(4)-\mathrm{N}(5)$ & $1.890(8)$ & $\mathrm{Mo}(8)-\mathrm{O}(33)$ & $1.834(6)$ \\
\hline $\mathrm{Cu}(4)-\mathrm{N}(3)$ & $1.907(8)$ & $\mathrm{Mo}(8)-\mathrm{O}(63)$ & $1.856(6)$ \\
\hline $\mathrm{Cu}(5)-\mathrm{N}(1)$ & $1.911(10)$ & $\mathrm{Mo}(8)-\mathrm{O}(23)$ & $2.067(6)$ \\
\hline $\mathrm{Cu}(5)-\mathrm{N}(2)$ & $1.925(9)$ & $\mathrm{Mo}(8)-\mathrm{O}(43)$ & $2.071(6)$ \\
\hline $\mathrm{Mo}(1)-\mathrm{O}(53)$ & $1.672(6)$ & $\mathrm{Mo}(8)-\mathrm{O}(30)$ & $2.418(6)$ \\
\hline $\mathrm{Mo}(1)-\mathrm{O}(60)$ & $1.921(6)$ & $\mathrm{Mo}(9)-\mathrm{O}(18)$ & $1.683(6)$ \\
\hline $\mathrm{Mo}(1)-\mathrm{O}(68)$ & $1.928(6)$ & $\mathrm{Mo}(9)-\mathrm{O}(56)$ & $1.843(6)$ \\
\hline $\mathrm{Mo}(1)-\mathrm{O}(50)$ & $1.944(6)$ & $\mathrm{Mo}(9)-\mathrm{O}(70)$ & $1.845(6)$ \\
\hline $\mathrm{Mo}(1)-\mathrm{O}(54)$ & $1.963(6)$ & $\mathrm{Mo}(9)-\mathrm{O}(23)$ & $2.050(6)$ \\
\hline $\mathrm{Mo}(1)-\mathrm{O}(31)$ & $2.409(6)$ & $\mathrm{Mo}(9)-\mathrm{O}(71)$ & $2.061(6)$ \\
\hline $\mathrm{Mo}(2)-\mathrm{O}(20)$ & $1.649(6)$ & $\mathrm{Mo}(9)-\mathrm{O}(30)$ & $2.394(6)$ \\
\hline $\mathrm{Mo}(2)-\mathrm{O}(33)$ & $1.947(6)$ & $\mathrm{Mo}(10)-\mathrm{O}(26)$ & $1.691(6)$ \\
\hline $\mathrm{Mo}(2)-\mathrm{O}(1)$ & $1.954(6)$ & $\mathrm{Mo}(10)-\mathrm{O}(47)$ & $1.842(6)$ \\
\hline $\mathrm{Mo}(2)-\mathrm{O}(56)$ & $1.957(6)$ & $\mathrm{Mo}(10)-\mathrm{O}(60)$ & $1.852(6)$ \\
\hline $\mathrm{Mo}(2)-\mathrm{O}(58)$ & $1.966(6)$ & $\mathrm{Mo}(10)-\mathrm{O}(61)$ & $1.997(6)$ \\
\hline $\mathrm{Mo}(2)-\mathrm{O}(30)$ & $2.413(6)$ & $\mathrm{Mo}(10)-\mathrm{O}(66)$ & $2.060(6)$ \\
\hline $\mathrm{Mo}(3)-\mathrm{O}(52)$ & $1.622(6)$ & $\mathrm{Mo}(10)-\mathrm{O}(65)$ & $2.409(6)$ \\
\hline $\mathrm{Mo}(3)-\mathrm{O}(46)$ & $1.917(6)$ & $\mathrm{Mo}(11)-\mathrm{O}(45)$ & $1.706(6)$ \\
\hline $\mathrm{Mo}(3)-\mathrm{O}(63)$ & $1.919(6)$ & $\mathrm{Mo}(11)-\mathrm{O}(1)$ & $1.828(6)$ \\
\hline $\mathrm{Mo}(3)-\mathrm{O}(47)$ & $1.969(6)$ & $\mathrm{Mo}(11)-\mathrm{O}(67)$ & $1.850(6)$ \\
\hline $\mathrm{Mo}(3)-\mathrm{O}(67)$ & $1.978(6)$ & $\mathrm{Mo}(11)-\mathrm{O}(57)$ & $2.008(6)$ \\
\hline $\mathrm{Mo}(3)-\mathrm{O}(65)$ & $2.355(6)$ & $\mathrm{Mo}(11)-\mathrm{O}(66)$ & $2.058(6)$ \\
\hline $\mathrm{Mo}(4)-\mathrm{O}(15)$ & $1.703(6)$ & $\mathrm{Mo}(11)-\mathrm{O}(65)$ & $2.332(6)$ \\
\hline $\mathrm{Mo}(4)-\mathrm{O}(58)$ & $1.827(6)$ & $\mathrm{Mo}(12)-\mathrm{O}(25)$ & $1.705(6)$ \\
\hline $\mathrm{Mo}(4)-\mathrm{O}(7)$ & $1.838(6)$ & $\mathrm{Mo}(12)-\mathrm{O}(69)$ & $1.827(6)$ \\
\hline $\mathrm{Mo}(4)-\mathrm{O}(57)$ & $2.037(6)$ & $\mathrm{Mo}(12)-\mathrm{O}(50)$ & $1.868(6)$ \\
\hline $\mathrm{Mo}(4)-\mathrm{O}(74)$ & $2.074(6)$ & $\mathrm{Mo}(12)-\mathrm{O}(71)$ & $2.039(6)$ \\
\hline $\mathrm{Mo}(4)-\mathrm{O}(01)$ & $2.354(6)$ & $\mathrm{Mo}(12)-\mathrm{O}(55)$ & $2.056(6)$ \\
\hline $\mathrm{Mo}(5)-\mathrm{O}(28)$ & $1.666(7)$ & $\mathrm{Mo}(12)-\mathrm{O}(31)$ & $2.382(6)$ \\
\hline $\mathrm{Mo}(5)-\mathrm{O}(70)$ & $1.937(6)$ & $\mathrm{V}(1)-\mathrm{O}(10)$ & $1.592(7)$ \\
\hline $\mathrm{Mo}(5)-\mathrm{O}(69)$ & $1.951(6)$ & $\mathrm{V}(1)-\mathrm{O}(71)$ & $1.906(6)$ \\
\hline
\end{tabular}




\begin{tabular}{|c|c|c|c|}
\hline $\mathrm{Mo}(5)-\mathrm{O}(72)$ & $1.953(6)$ & $\mathrm{V}(1)-\mathrm{O}(43)$ & $1.943(6$ \\
\hline $\mathrm{Mo}(5)-\mathrm{O}(7)$ & $1.956(7)$ & $\mathrm{V}(1)-\mathrm{O}(23)$ & $1.976(6$ \\
\hline $\mathrm{Mo}(5)-\mathrm{O}(01)$ & $2.375(6)$ & $\mathrm{V}(1)-\mathrm{O}(55)$ & $2.006(6$ \\
\hline $\mathrm{V}(2)-\mathrm{O}(74)$ & $2.022(6)$ & $\mathrm{V}(2)-\mathrm{O}(42)$ & $1.625(6$ \\
\hline $\mathrm{V}(2)-\mathrm{O}(61)$ & $2.067(6)$ & $\mathrm{V}(2)-\mathrm{O}(57)$ & $1.934(6$ \\
\hline $\mathrm{V}(2)-\mathrm{OW} 1$ & $2.220(6)$ & $\mathrm{V}(2)-\mathrm{O}(66)$ & $2.019(6$ \\
\hline $\mathrm{O}(30)-\mathrm{As}(1)-\mathrm{O}(65)$ & $108.6(3)$ & $\mathrm{O}(42)-\mathrm{V}(2)-\mathrm{O}(74)$ & $109.7(3$ \\
\hline $\mathrm{O}(30)-\mathrm{As}(1)-\mathrm{O}(01)$ & $108.4(3)$ & $\mathrm{O}(57)-\mathrm{V}(2)-\mathrm{O}(74)$ & $78.2(3)$ \\
\hline $\mathrm{O}(65)-\mathrm{As}(1)-\mathrm{O}(01)$ & $113.0(3)$ & $\mathrm{O}(66)-\mathrm{V}(2)-\mathrm{O}(74)$ & $138.3(3$ \\
\hline $\mathrm{O}(30)-\mathrm{As}(1)-\mathrm{O}(31)$ & $110.6(3)$ & $\mathrm{O}(42)-\mathrm{V}(2)-\mathrm{O}(61)$ & $160.3(3$ \\
\hline $\mathrm{O}(65)-\mathrm{As}(1)-\mathrm{O}(31)$ & $108.6(3)$ & $\mathrm{O}(57)-\mathrm{V}(2)-\mathrm{O}(61)$ & $98.6(2)$ \\
\hline $\mathrm{O}(01)-\mathrm{As}(1)-\mathrm{O}(31)$ & $107.7(3)$ & $\mathrm{O}(66)-\mathrm{V}(2)-\mathrm{O}(61)$ & $75.2(2)$ \\
\hline $\mathrm{N}(8)-\mathrm{Cu}(1)-\mathrm{N}(9)$ & $169.8(4)$ & $\mathrm{O}(74)-\mathrm{V}(2)-\mathrm{O}(61)$ & $74.9(2)$ \\
\hline $\mathrm{N}(8)-\mathrm{Cu}(1)-\mathrm{O}(45) \# 1$ & $93.5(3)$ & $\mathrm{O}(42)-\mathrm{V}(2)-\mathrm{OW} 1$ & $85.0(3)$ \\
\hline $\mathrm{N}(9)-\mathrm{Cu}(1)-\mathrm{O}(45) \# 1$ & $96.4(3)$ & $\mathrm{O}(57)-\mathrm{V}(2)-\mathrm{OW} 1$ & $173.9(3$ \\
\hline $\mathrm{N}(4)-\mathrm{Cu}(2)-\mathrm{N}(10)$ & $168.7(3)$ & $\mathrm{O}(66)-\mathrm{V}(2)-\mathrm{OW} 1$ & $100.4(2$ \\
\hline $\mathrm{N}(4)-\mathrm{Cu}(2)-\mathrm{O}(52)$ & $93.8(3)$ & $\mathrm{O}(74)-\mathrm{V}(2)-\mathrm{OW} 1$ & $99.5(2)$ \\
\hline $\mathrm{N}(10)-\mathrm{Cu}(2)-\mathrm{O}(52)$ & $97.5(3)$ & $\mathrm{O}(61)-\mathrm{V}(2)-\mathrm{OW} 1$ & $75.3(2)$ \\
\hline $\mathrm{N}(7)-\mathrm{Cu}(3)-\mathrm{N}(6)$ & $160.4(4)$ & $\mathrm{O}(43)-\mathrm{V}(1)-\mathrm{O}(23)$ & $80.1(3)$ \\
\hline $\mathrm{N}(7)-\mathrm{Cu}(3)-\mathrm{O}(42)$ & $101.3(3)$ & $\mathrm{O}(10)-\mathrm{V}(1)-\mathrm{O}(55)$ & $105.6(3$ \\
\hline $\mathrm{N}(6)-\mathrm{Cu}(3)-\mathrm{O}(42)$ & $98.3(3)$ & $\mathrm{O}(71)-\mathrm{V}(1)-\mathrm{O}(55)$ & $80.3(3)$ \\
\hline $\mathrm{N}(5)-\mathrm{Cu}(4)-\mathrm{N}(3)$ & $170.4(3)$ & $\mathrm{O}(43)-\mathrm{V}(1)-\mathrm{O}(55)$ & $79.1(3)$ \\
\hline $\mathrm{N}(1)-\mathrm{Cu}(5)-\mathrm{N}(2)$ & $168.2(4)$ & $\mathrm{O}(23)-\mathrm{V}(1)-\mathrm{O}(55)$ & $142.2(3$ \\
\hline $\mathrm{O}(10)-\mathrm{V}(1)-\mathrm{O}(71)$ & $123.9(4)$ & $\mathrm{O}(42)-\mathrm{V}(2)-\mathrm{O}(57)$ & $101.1(3$ \\
\hline $\mathrm{O}(10)-\mathrm{V}(1)-\mathrm{O}(43)$ & $122.0(4)$ & $\mathrm{O}(42)-\mathrm{V}(2)-\mathrm{O}(66)$ & $108.3(3$ \\
\hline $\mathrm{O}(71)-\mathrm{V}(1)-\mathrm{O}(43)$ & $113.9(3)$ & $\mathrm{O}(57)-\mathrm{V}(2)-\mathrm{O}(66)$ & $78.2(2)$ \\
\hline $\mathrm{O}(10)-\mathrm{V}(1)-\mathrm{O}(23)$ & $112.2(3)$ & $\mathrm{O}(71)-\mathrm{V}(1)-\mathrm{O}(23)$ & $79.9(3)$ \\
\hline
\end{tabular}

Symmetry transformations used to generate equivalent atoms: \#1 $x+1 / 2,-y+3 / 2, z+1 / 2$; \#2 $\mathrm{x}-1 / 2,-\mathrm{y}+3 / 2, \mathrm{z}-1 / 2$; \#3 -x,-y+2,-z ; \#4 -x+1/2,y+1/2,-z+1/2 ; \#5 -x+1/2,y-1/2,-z+1/2 ; \#6 -x-1,-y+2,-z; \#7 -x,-y+1,-z ; \#8 -x+1/2,y-1/2,-z-1/2 ; \#9 -x+1/2,y+1/2,-z-1/2 ; \#10 -x-1,-y+1,-z.. 
Table S2 Selected bond lengths ( $\AA$ ) and bond angles (deg) for compound 2.

\begin{tabular}{|c|c|c|c|}
\hline $\mathrm{As}(1)-\mathrm{O}(14) \# 1$ & $1.691(17)$ & $\mathrm{Mo}(4)-\mathrm{O}(4)$ & $1.648(9)$ \\
\hline $\operatorname{As}(1)-\mathrm{O}(14)$ & $1.691(17)$ & $\mathrm{Mo}(4)-\mathrm{O}(17)$ & $1.847(11)$ \\
\hline $\mathrm{As}(1)-\mathrm{O}(22)$ & $1.720(14)$ & $\mathrm{Mo}(4)-\mathrm{O}(8)$ & $1.894(12)$ \\
\hline $\mathrm{As}(1)-\mathrm{O}(22) \# 1$ & $1.720(14)$ & $\mathrm{Mo}(4)-\mathrm{O}(11)$ & $1.944(10)$ \\
\hline $\mathrm{As}(1)-\mathrm{O}(1) \# 1$ & $1.756(13)$ & $\mathrm{Mo}(4)-\mathrm{O}(12)$ & $1.959(10)$ \\
\hline $\operatorname{As}(1)-O(1)$ & $1.756(13)$ & $\mathrm{Mo}(4)-\mathrm{O}(1)$ & $2.375(13)$ \\
\hline $\mathrm{Mo}(1)-\mathrm{O}(16)$ & $1.677(9)$ & $\mathrm{Mo}(4)-\mathrm{O}(22)$ & $2.415(15)$ \\
\hline $\mathrm{Mo}(1)-\mathrm{O}(9)$ & $1.856(10)$ & $\mathrm{Mo}(5)-\mathrm{O}(15)$ & $1.605(9)$ \\
\hline $\mathrm{Mo}(1)-\mathrm{O}(21)$ & $1.873(11)$ & $\mathrm{Mo}(5)-\mathrm{O}(8)$ & $1.875(10)$ \\
\hline $\mathrm{Mo}(1)-\mathrm{O}(3)$ & $1.900(10)$ & $\mathrm{Mo}(5)-\mathrm{O}(10)$ & $1.898(11)$ \\
\hline $\mathrm{Mo}(1)-\mathrm{O}(11) \# 1$ & $1.961(10)$ & $\mathrm{Mo}(5)-\mathrm{O}(19) \# 1$ & $1.908(10)$ \\
\hline $\mathrm{Mo}(1)-\mathrm{O}(1) \# 1$ & $2.324(12)$ & $\mathrm{Mo}(5)-\mathrm{O}(9)$ & $1.961(9)$ \\
\hline $\mathrm{Mo}(1)-\mathrm{O}(2) \# 1$ & $2.492(15)$ & $\mathrm{Mo}(5)-\mathrm{O}(22)$ & $2.343(12)$ \\
\hline $\mathrm{Mo}(2)-\mathrm{O}(5)$ & $1.656(9)$ & $\mathrm{Mo}(5)-\mathrm{O}(2) \# 1$ & $2.471(14)$ \\
\hline $\operatorname{Mo}(2)-\mathrm{O}(13)$ & $1.850(10)$ & $\mathrm{Mo}(6)-\mathrm{O}(6)$ & $1.667(9)$ \\
\hline $\operatorname{Mo}(2)-\mathrm{O}(10)$ & $1.854(10)$ & $\mathrm{Mo}(6)-\mathrm{O}(19)$ & $1.871(13)$ \\
\hline $\mathrm{Mo}(2)-\mathrm{O}(18)$ & $1.967(10)$ & $\mathrm{Mo}(6)-\mathrm{O}(12) \# 1$ & $1.904(10)$ \\
\hline $\operatorname{Mo}(2)-\mathrm{O}(3)$ & $1.984(9)$ & $\mathrm{Mo}(6)-\mathrm{O}(20)$ & $1.912(10)$ \\
\hline $\mathrm{Mo}(2)-\mathrm{O}(14)$ & $2.331(14)$ & $\mathrm{Mo}(6)-\mathrm{O}(18)$ & $1.911(11)$ \\
\hline $\mathrm{Mo}(2)-\mathrm{O}(2) \# 1$ & $2.435(17)$ & $\mathrm{Mo}(6)-\mathrm{O}(22) \# 1$ & $2.405(15)$ \\
\hline $\mathrm{Mo}(3)-\mathrm{O}(7)$ & $1.627(9)$ & $\mathrm{Mo}(6)-\mathrm{O}(14)$ & $2.409(16)$ \\
\hline $\mathrm{Mo}(3)-\mathrm{O}(20)$ & $1.853(11)$ & $\mathrm{V}-\mathrm{O}(23)$ & $1.57(2)$ \\
\hline $\mathrm{Mo}(3)-\mathrm{O}(17)$ & $1.919(11)$ & $\mathrm{V}-\mathrm{O}(11)$ & $1.804(14)$ \\
\hline $\mathrm{Mo}(3)-\mathrm{O}(21) \# 1$ & $1.920(13)$ & $\mathrm{V}-\mathrm{O}(3) \# 1$ & $1.830(12)$ \\
\hline $\mathrm{Mo}(3)-\mathrm{O}(13)$ & $1.935(11)$ & $\mathrm{V}-\mathrm{O}(18) \# 1$ & $2.045(13)$ \\
\hline $\mathrm{Mo}(3)-\mathrm{O}(1)$ & $2.397(13)$ & $\mathrm{V}-\mathrm{O}(12)$ & $2.045(14)$ \\
\hline $\mathrm{Mo}(3)-\mathrm{O}(14)$ & $2.446(14)$ & $\mathrm{Cu}(1)-\mathrm{N}(3)$ & $1.873(9)$ \\
\hline $\mathrm{Cu}(2)-\mathrm{N}(4)$ & $1.874(9)$ & $\mathrm{Cu}(1)-\mathrm{N}(1) \# 2$ & $1.880(10)$ \\
\hline $\mathrm{Cu}(2)-\mathrm{N}(4) \# 3$ & $1.874(9)$ & $\mathrm{O}(22) \# 1-\mathrm{As}(1)-\mathrm{O}(1)$ & $108.1(6)$ \\
\hline $\mathrm{O}(2) \# 1-\mathrm{As}(1)-\mathrm{O}(2)$ & $180.000(2)$ & $\mathrm{O}(1) \# 1-\mathrm{As}(1)-\mathrm{O}(1)$ & $180.0(7)$ \\
\hline $\mathrm{O}(2) \# 1-\mathrm{As}(1)-\mathrm{O}(14) \# 1$ & $112.7(8)$ & $\mathrm{O}(23)-\mathrm{V}-\mathrm{O}(11)$ & $109.7(12)$ \\
\hline $\mathrm{O}(2)-\mathrm{As}(1)-\mathrm{O}(14) \# 1$ & $67.3(8)$ & $\mathrm{O}(23)-\mathrm{V}-\mathrm{O}(3) \# 1$ & $106.0(12)$ \\
\hline $\mathrm{O}(14) \# 1-\mathrm{As}(1)-\mathrm{O}(14)$ & $180.000(2)$ & $\mathrm{O}(11)-\mathrm{V}-\mathrm{O}(3) \# 1$ & $86.2(5)$ \\
\hline $\mathrm{O}(2) \# 1-\mathrm{As}(1)-\mathrm{O}(22)$ & $68.4(7)$ & $\mathrm{O}(23)-\mathrm{V}-\mathrm{O}(18) \# 1$ & $113.4(11)$ \\
\hline $\mathrm{O}(2)-\mathrm{As}(1)-\mathrm{O}(22)$ & $111.6(7)$ & $\mathrm{O}(11)-\mathrm{V}-\mathrm{O}(18) \# 1$ & $136.9(8)$ \\
\hline $\mathrm{O}(14) \# 1-\mathrm{As}(1)-\mathrm{O}(22)$ & $72.5(7)$ & $\mathrm{O}(3) \# 1-\mathrm{V}-\mathrm{O}(18) \# 1$ & $82.3(5)$ \\
\hline $\mathrm{O}(14)-\mathrm{As}(1)-\mathrm{O}(22)$ & $107.5(7)$ & $\mathrm{O}(23)-\mathrm{V}-\mathrm{O}(12)$ & $118.8(12)$ \\
\hline $\mathrm{O}(2) \# 1-\mathrm{As}(1)-\mathrm{O}(22) \# 1$ & $111.6(7)$ & $\mathrm{O}(11)-\mathrm{V}-\mathrm{O}(12)$ & $82.6(5)$ \\
\hline $\mathrm{O}(2) \# 1-\mathrm{A} s(1)-\mathrm{O}(1) \# 1$ & $69.3(7)$ & $\mathrm{O}(3) \# 1-\mathrm{V}-\mathrm{O}(12)$ & $135.0(7)$ \\
\hline $\mathrm{O}(2)-\mathrm{As}(1)-\mathrm{O}(1) \# 1$ & $110.7(7)$ & $\mathrm{O}(18) \# 1-\mathrm{V}-\mathrm{O}(12)$ & $77.0(5)$ \\
\hline $\mathrm{O}(14) \# 1-\mathrm{As}(1)-\mathrm{O}(1) \# 1$ & $74.1(7)$ & $\mathrm{O}(22)-\mathrm{As}(1)-\mathrm{O}(1) \# 1$ & $108.1(6)$ \\
\hline $\mathrm{O}(14)-\mathrm{As}(1)-\mathrm{O}(1) \# 1$ & $105.9(6)$ & $\mathrm{O}(22) \# 1-\mathrm{As}(1)-\mathrm{O}(1) \# 1$ & $71.9(6)$ \\
\hline
\end{tabular}

Symmetry transformations used to generate equivalent atoms: \#1 -x+1,-y,-z+1; \#2 x,y,z-1; \#3 $-\mathrm{x},-\mathrm{y}+1,-\mathrm{z}+1 ; \# 4 \mathrm{x}, \mathrm{y}, \mathrm{z}+1 ; \# 5-\mathrm{x},-\mathrm{y}+1,-\mathrm{z}+2 ; \# 6-\mathrm{x}+2,-\mathrm{y}+2,-\mathrm{z}$. 